\title{
Effect of fish oil on neutrophil chemiluminescence induced by different stimuli in patients with rheumatoid arthritis
}

\author{
M Magarò, A Zoli, L Altomonte, L Mirone, P De Sole, G Di Mario, E De Leo
}

\begin{abstract}
Lipid composition plays an important part in the structural and metabolic functions of cell membranes. In particular the production of inflammatory mediators such as prostaglandins and leukotrienes is dependent on polyunsaturated fatty acid precursors. Neutrophil leucocytes participate in inflammatory processes by their phagocytic and killing activities which can be monitored by measuring the photon emission (chemiluminescence).

Chemiluminescence was measured in a luminol dependent system after stimulation by either particulate (zymosan) or soluble (phorbol myristate acetate) stimulus in a group of 10 patients with rheumatoid arthritis before and 21 and 45 days after treatment with a diet supplemented with eicosapentaenoic and docosahexaenoic acids. Ten patients with rheumatoid arthritis continuing their usual diet were used as control subjects. A progressive reduction of chemiluminescence stimulated by zymosan and phorbol myristate acetate was found in the patients treated with fish oil supplementation. This result correlated well with the reduction in erythrocyte sedimentation rate and an improvement of clinical parameters.
\end{abstract}

The effects of fish oil derived lipids on neutrophil chemiluminescence are probably due to a change of the lipid composition of the cell membrane which is dependent on the esterification of eicosapentaenoic acid and docosahexaenoic acid in cellular membrane phospholipids. The modification of membrane lipid composition seems to interact in a nonspecific way with the metabolic activation of neutrophils during phagocytosis.

(Ann Rheum Dis 1992; 51: 877-880)

Alterations in dietary lipid composition have been shown to cause major changes in the synthesis of lipid derived mediators of inflammation, especially eicosanoids derived from arachidonic acid, ${ }^{1}$ leading to the suggestion that the modulation of dietary fatty acids can alter inflammatory responses in rheumatic diseases. ${ }^{2}$

Although the most common prostaglandins and leukotrienes are derived from arachidonic acid, other polyunsaturated fatty acids may give rise to homologous compounds. Diets containing $\mathrm{n}-3$ fatty acids, mainly eicosapentaenoic acid (C 20:5) and docosahexaenoic acid (C 22:6) usually induce low levels of plasma arachidonic acid (C 20:4). Moreover eicosapentaenoic acid and docosahexaenoic acid interfere in a competitive way with the cyclo-oxygenase and lipo-oxygenase pathway of arachidonic acid metabolism and therefore generate oxidised products that are less inflammatory than those derived from arachidonic acid metabolism. ${ }^{3-7}$

Neutrophil leucocytes actively participate in inflammatory processes by means of their phagocytic and killing activities that can be monitored by measuring the photon emission (chemiluminescence) directly related to activation of NADPH oxidase and myeloperoxidase activity. ${ }^{8}$ The activation of membrane NADPH oxidase is preceded by and mediated through a modification of membrane bound phospholipase $\mathrm{C}$ or the translocation-activation of protein kinase $\mathrm{C}$ from cytosol to plasma membrane. Different stimuli such as opsonised zymosan or phorbol myristate acetate can exert their action on the phospholipase C step through interaction with guanosine triphosphate binding protein or can directly induce the translocation-activation of protein kinase C. ${ }^{10}$

Membrane lipid composition plays a fundamental part in the neutrophil cell activation process. An adequate dietary supply of fish derived oil, which is the main source of eicosapentaenoic acid and docosahexaenoic acid, was effective in reducing whole blood chemiluminescence induced by opsonised zymosan in a group of patients with rheumatoid arthritis (RA). ${ }^{11}$

In this study we examined the effect of eicosapentaenoic acid and docosahexaenoic acid on neutrophil function determined by whole blood chemiluminescence induced by stimuli which differ in their neutrophil activation pattern, in patients with RA. The chemiluminescence results were compared with clinical and laboratory parameters.

\section{Patients and methods}

Twenty women with RA as defined by the American Rheumatism Association 1987 revised criteria $^{12}$ gave their informed consent to the study. The patients, aged between 25 and 45 years, were not obese, not diabetic, and were non-smokers. All patients had active disease as defined by the following criteria: morning stiffness of at least 30 minutes duration, three or more swollen joints, six or more tender joints, and an erythrocyte sedimentation rate of at least $30 \mathrm{~mm}$ in the first hour.

The patients were not treated with systemic steroids, immunosuppressive, nor disease modifying drugs in the three months before the beginning of the study. They were randomly divided into two groups. Ten patients (group A) supplemented their usual diet with nine $1 \mathrm{~g}$ capsules of Maxepa (Ciba Geigy) for 45 days. 
Maxepa consists almost entirely of triglycerides of which eicosapentaenoic acid and docosahexaenoic acid constitute $34 \%$ of the total fatty acids and $86 \%$ of polyunsaturated fatty acids; the arachidonic precursor, linoleic acid, constitutes less than $2 \%$ of the fatty acids and arachidonic acid is not detectable. A total of $1.6 \mathrm{~g}$ of eicosapentaenoic acid and $1.1 \mathrm{~g}$ of docosahexaenoic acid (339 kJ) were provided daily from this preparation. Ten patients (group B) continued their usual diet.

All patients were receiving stable doses of non-steroidal anti-inflammatory drugs (NSAIDs) before and during the study (diclofenac sodium, $100 \mathrm{mg} /$ day). Each patient was assessed before enrolling in the study and after 21 and 45 days. Clinical evaluation included duration of morning stiffness, grip strength measured as a mean of three readings from each of the right and left hands, and the Ritchie index.

At each assessment fasting blood samples were collected from the antecubital veins of patients to determine neutrophil chemiluminescence and for routine laboratory assessment.

Zymosan was prepared and opsonised as described previously.

Phorbol myristate acetate (Sigma, St Louis, MO, USA) was dissolved in dimethylsulphoxide to give a $3 \mathrm{mM}$ solution and stored at $-20^{\circ} \mathrm{C}$. A working solution in a modified Krebs Ringer medium containing $5.5 \mathrm{mM}$ glucose and 0.4 $\mathrm{mM} \mathrm{CaCl} 2$ was prepared just before use.

Chemiluminescence was determined in a luminol amplified whole blood system according to a modification of a previously reported method. ${ }^{9}$ Briefly, $0.5 \mu \mathrm{l}$ of EDTA anticoagulated blood, $100 \mathrm{nmol}$ luminol and $0.5 \mathrm{mg}$ opsonised zymosan or $150 \mathrm{pmol}$ PMA were added to a $1 \mathrm{ml}$ final volume of modified Krebs Ringer medium. Chemiluminescence was measured at $25^{\circ} \mathrm{C}$ in an automatic luminometer (Picolite 6500, Packard Instruments) for two hours at five minute cycles. The specific activity was calculated by the ratio of maximum chemiluminescence activity (counts/s) over the number of neutrophils in the test vial.

Results are expressed as mean (SD). The Kolmorogov-Smirnov test was used to assess the data distribution, which was found to be normal in the groups studied. Statistical analysis was performed by ANOVA and Student's $t$ test.

\section{Results}

No significant differences in the clinical variables were observed between the two groups of patients at the beginning of the study. A comparison of the clinical measures of disease activity after 45 days with those obtained on entry to the study showed a significant statistical decrease in the Ritchie index $(p<0.05)$, in morning stiffness $(p<0.05)$, and a significant increase in grip strength $(p<0.05)$ in group A patients (table 1). In group B patients (control group) a tendency towards benefit was observed at the end of the study but this was not significant compared with basal values (table 2 ).

Figures 1 and 2 show chemiluminescence specific activities stimulated by particulate (zymosan) or soluble (phorbol myristate acetate) stimuli in patients treated with Maxepa and in control subjects.

Group A patients showed a progressive reduction of neutrophil chemiluminescence stimulated by zymosan that was statistically significant at the end of the study $(6.02(2.89) v$ $7.7(2.69) ; \mathrm{p}<0.05)$ and a more remarkable decrease of neutrophil chemiluminescence stimulated by phorbol myristate acetate that was statistically significant at 21 days $(5 \cdot 19(2 \cdot 5)$ $v 7.01(3.31) ; \mathrm{p}<0.02)$ and at 45 days $(5.08$

Table 1 Clinical and laboratory parameters in group $A$ patients. Results are given as mean $(S D)$ values. $p<0.05$ in all instances

\begin{tabular}{lcc}
\hline Parameter & \multicolumn{2}{l}{ Time (days) } \\
\cline { 2 - 3 } & 0 & 45 \\
\hline Ritchie index & $21 \cdot 1(7 \cdot 4)$ & $9(4)$ \\
Grip strength (mmHg) & $53 \cdot 9(17 \cdot 5)$ & $87 \cdot 1(26 \cdot 4)$ \\
Morning stiffness (min) & $43 \cdot 2(20 \cdot 9)$ & $20 \cdot 9(16 \cdot 9)$ \\
Pain (visual analogue scale) (cm) & $7 \cdot 1(1 \cdot 9)$ & $4 \cdot 8(1 \cdot 2)$ \\
Erythrocyte sedimentation & & \\
rate (mm/lst hour) & $77 \cdot 2(42 \cdot 8)$ & $59 \cdot 5(39 \cdot 2)$ \\
Serum IgG (g/l) & $22 \cdot 0(6 \cdot 1)$ & $20 \cdot 1(5 \cdot 6)$ \\
\hline
\end{tabular}

Table 2 Clinical and laboratory parameters in group $B$ patients. Results are given as mean (SD) values. The p value was not significant in all instances

\begin{tabular}{lcc}
\hline Parameter & \multicolumn{2}{l}{ Time (days) } \\
\cline { 2 - 3 } & 0 & 45 \\
\hline Ritchie index & $16 \cdot 6(4 \cdot 5)$ & $12 \cdot 8(6 \cdot 9)$ \\
Grip strength (mmHg) & $61 \cdot 6(20 \cdot 6)$ & $91(40)$ \\
Morning stiffness (min) & $21 \cdot 0(20 \cdot 1)$ & $19(12)$ \\
Pain (visual analogue scale) (cm) & $5 \cdot 2(1 \cdot 7)$ & $4 \cdot 2(1 \cdot 6)$ \\
Erythrocyte sedimentation & $66(34 \cdot 1)$ & $66(38 \cdot 8)$ \\
$\quad$ rate (mm/lst hour) & $15 \cdot 7(3 \cdot 3)$ & $16 \cdot 0(3 \cdot 7)$ \\
Serum IgG $(\mathrm{g} / \mathrm{l})$ & &
\end{tabular}
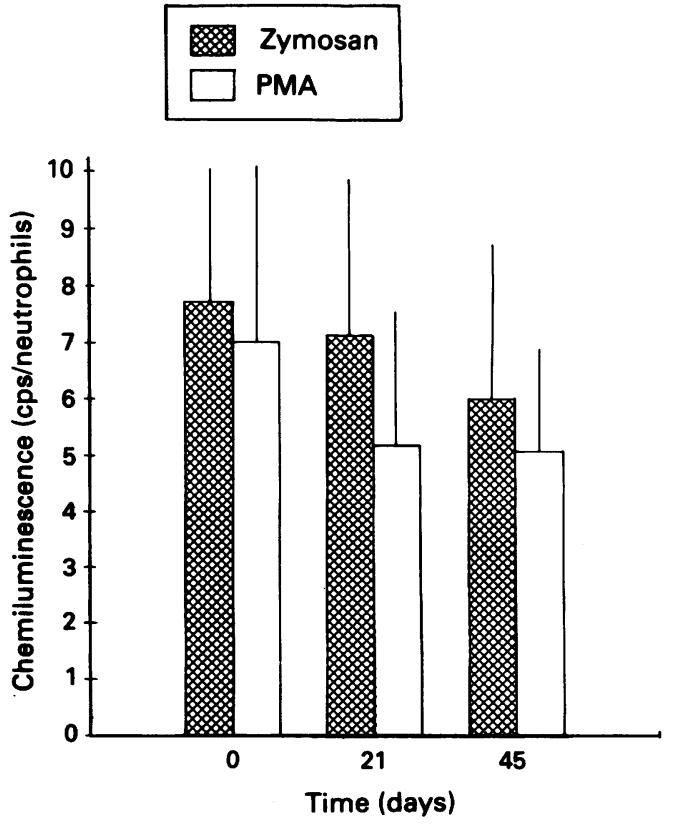

Figurel Neutrophilchemiluminescence(obtained by dividing the maximum chemiluminescence activity by the number of neutrophils) stimulated by zymosan or phorbol myristate acetate $(P M A)$ at the start of treatment and after 21 and 45 days in group $A$ patients. 


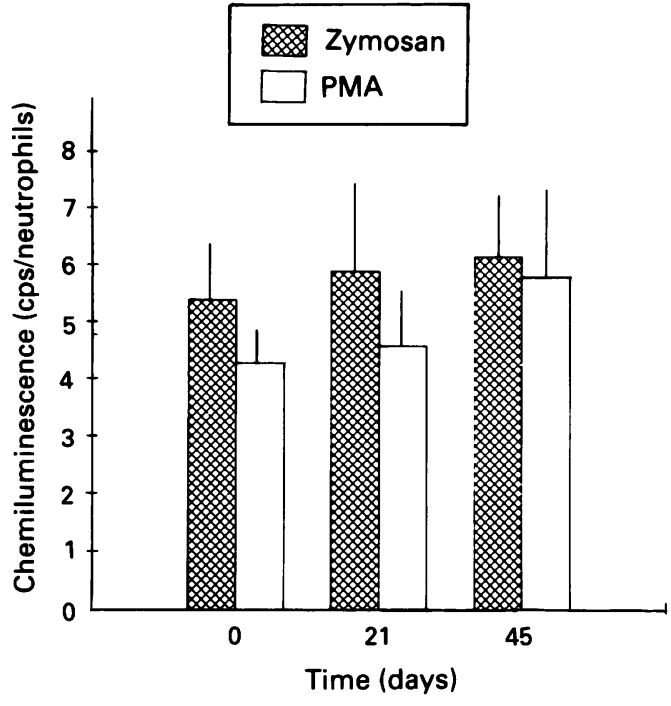

Figure 2 Neutrophilchemiluminescence(obtained by dividing the maximum chemiluminescence activity by the number of neutrophils) stimulated by zymosan or phorbol myristate acetate (PMA) at the start of treatment and after 21 and 45 days in group $B$ patients.

(1.93) v 7.01 (3.31); $\mathrm{p}<0.02)$ compared with basal values.

In control subjects there was no difference in chemiluminescence with respect to basal values for zymosan stimulation, whereas for phorbol myristate acetate stimulation an increase in activity was obtained (fig 2).

The erythrocyte sedimentation rate and serum IgG levels were decreased at the end of the study compared with the initial values in group A patients (table 1).

\section{Discussion}

Lipid composition is a main factor of the structure and biological function of cell membranes. The presence and location of even one double bond may be sufficient to exert a profound influence on the physicochemical properties of cell membranes. In particular the fluidity of membranes and the gel-liquid phase transition temperature are strictly dependent on fatty acid and phospholipid composition. Membrane bound enzyme activity and receptor number or function are directly dependent on the lipid composition.

Dietary polyunsaturated fatty acids, including arachidonic acid, eicosapentaenoic acid, and docosahexaenoic acid, are esterified in cellular membrane phospholipids. ${ }^{13}$ On cell activation phospholipase $A_{2}$ is activated resulting in the release of unesterified fatty acids. The arachidonic acid, eicosapentaenoic acid, and docosahexaenoic acid released can serve as substrates for the cyclo-oxygenase and 5-lipooxygenase pathways. The incorporation of $n-3$ fatty acids into cellular membranes reduces the availability of arachidonic acid by cyclooxygenase $^{34}$ and alters the formation of leukotrienes by stimulated human leucocytes. ${ }^{13}$

In a previous investigation we showed a reduced neutrophil chemiluminescence depending on particulate stimulus in patients with RA treated with a diet rich in polyunsaturated fatty acids supplemented with eicosapentaenoic acid and docosahexaenoic acid. ${ }^{11}$ This work corroborates the finding of a reduced neutrophil chemiluminescence in patients with active RA treated with a diet rich in marine lipids. The decreased neutrophil chemiluminescence is not dependent on the physical and chemical characteristics of the stimulus used as it was found to be reduced either with particulate or with soluble stimuli.

The reduction of neutrophil enzymatic activities in these patients seems to be related to an alteration of the physicochemical properties of neutrophil cell membranes induced by eicosapentaenoic acid and docosahexaenoic acid. NADPH oxidase activation, which is primarily responsible for chemiluminescence production by polymorphonuclear cells is due to different, although related, mechanisms. In particular phorbol esters and opsonised zymosan differ in their ability to induce NADPH oxidase activation in polymorphonuclear cells. ${ }^{10}$

Neutrophil cell activation processes highlight the role of plasma membrane lipids in polymorphonuclear cell function. The ligand binding to the extracellular side of a receptor and the following transmission of information through the membrane into the cytoplasmic side are greatly affected by the membrane lipid composition and related fluidity. The fluidity of the membrane is influenced by any modification of the degree of unsaturation or chain length, or both, of membrane fatty acids.

Both phorbol myristate acetate and opsonised zymosan stimulate the polymorphonuclear cells by a rearrangement of plasma membrane lipids. Phorbol myristate acetate acts directly through translocation-activation of protein kinase $\mathrm{C}$ from cytosol to plasma membrane. The activated protein kinase $\mathrm{C}$ system phosphorylates a 32 kilodalton protein which seems to be the reaction responsible for the enzymatic activation. ${ }^{14} 15$ Opsonised zymosan interacts with plasma membrane bound receptors that are coupled to guanine nucleotide binding regulatory proteins known as $\mathrm{G}$ proteins. ${ }^{16}$ Guanosine triphosphate binding proteins induce a phospholipase $\mathrm{C}$ mediated release of inositol triphosphate and diacylglycerol. The mobilisation of intracellular calcium ions induced by inositol triphosphate and diacylglycerol is responsible for the kinase activation. ${ }^{17}$

As zymosan and phorbol dependent chemiluminescence were reduced in response to the diet rich in eicosapentaenoic acid and docosahexaenoic acid, it seems probable that the alteration of membrane lipid composition modifies neutrophil metabolic activation during phagocytosis in a non-specific way. Neutrophil chemiluminescence induced by phorbol myristate acetate, a liposoluble stimulus which diffuses through cell membranes and depends highly on cell membrane fluidity, appears to be decreased earlier than chemiluminescence induced by zymosan, a particulate stimulus which interacts with cell receptors.

This study confirms our finding of a progressive clinical improvement in patients with RA treated with a diet rich in eicosapentaenoic acid and docosahexaenoic acid. ${ }^{11}$ The favourable 
effect observed with a diet rich in polyunsaturated fatty acids derived from fish oil is in agreement with the results of Kremer et al ${ }^{18}$ who observed an improvement in the number of tender joints, and those of Sperling et al ${ }^{19}$ who noted improvements in the joint pain index and the patient's assessment of disease activity after 14 and six weeks respectively of daily treatment with fish oil: Van der Tempel et al ${ }^{20}$ found an improvement in the joint swelling index and duration of morning stiffness after 12 weeks of treatment with $2.04 \mathrm{~g}$ eicosapentaenoic acid and $1.32 \mathrm{~g}$ docosahexaenoic acid daily.

Products formed from eicosapentaenoic acid show biological activities that are different from those shown by products formed from arachidonic acid. The ingestion of omega- 3 fatty acids provides a substrate for the production of the three series of prostaglandins and the five series of leukotrienes. Thromboxane $\mathrm{A}_{3}$ does not provoke the aggregation of platelets as does thromboxane $A_{2}{ }^{5}$ Similarly it has been pointed out that leukotriene $B_{5}$, derived from eicosapentaenoic acid, is considerably less active than leukotriene $B_{4}$ with respect to its effect on neutrophils. ${ }^{21} 22$

Studies of stimulated peripheral blood neutrophils of human volunteers ingesting marine lipids showed the inhibition of several inflammatory functions. $\mathrm{Lee}^{23}$ reported that human neutrophils stimulated in vitro with $10 \mu \mathrm{mol} / \mathrm{l}$ calcium ionophore A23187 in the presence of exogenous unesterified eicosapentaenoic acid generate a combination of leukotriene $\mathrm{B}_{4}$ and leukotriene $\mathrm{B}_{5}$ with a significant and selective decrease in arachidonic acid derived leukotriene $\mathrm{B}_{4}$ generation. This finding indicates the inhibition of the leukotriene $A$ epoxide hydrolase. Leukotriene $\mathrm{B}_{4}$ generation by neutrophils and monocytes stimulated in vitro with calcium ionophore was inhibited after six weeks of dietary supplementation with $18 \mathrm{~g}$ Maxepa daily. ${ }^{2}$ In patients with RA, the supplementation of diet with $20 \mathrm{~g}$ Maxepa daily induced a decreased ratio of arachidonic acid to eicosapentaenoic acid in neutrophil cellular lipids from $81: 1$ to $2 \cdot 7: 1$ and a significant suppression of leukotriene $B_{4}$ generation from the calcium ionophore stimulated neutrophils. ${ }^{19}$ In the study of Van der Temple et al ${ }^{20}$ neutrophil leukotriene $B_{4}$ production in vitro showed a reduction after 12 weeks of fish oil supplementation in patients with RA whereas leukotriene $B_{5}$ production increased from undetectable to substantial amounts.

Hostmark et al ${ }^{24}$ found a significant decrease of plasma fibrinogen, which is an acute phase protein recognised as being a white blood cell activator, during a six week daily supplement of 14 fish oil capsules in healthy volunteers. Our study shows a significant reduction in laboratory indexes of acute inflammation such as erythrocyte sedimentation rate and immunoglobulins.

In conclusion, dietary supplementation of n-3 fatty acids in patients with RA gives a modification of neutrophil enzymatic activities which alters the production of potent lipid mediators of inflammation such as prostaglandins and leukotrienes. The inhibitory effects of fish oil derived lipids are probably due to a change in the lipid composition of the neutrophil cell membrane dependent on the esterification of eicosapentaenoic acid and docosahexaenoic acid cellular membrane phospholipids and, consequently, to a cell membrane stabilisation. The modification of membrane lipid composition seems to interact in a non-specific way with the metabolic activation of neutrophils during phagocytosis.

1 Lee T H, Arm J P. Prospects for modifying the allergic responses by fish oils. Clin Allergy 1986; 16: 89-100.

2 Lee T H, Hoover R L, Williams J D, et al. Effect of dietary enrichment with eicosapentaenoic and docosahexaenoic acids on in vitro neutrophil and monocyte leukotriene generation and neutrophil function. N Engl f Med 1985 312: 1217-24.

3 Lands W E M. The fate of polyunsaturated fatty acids. In Simopoulos A P, Kifer R R, Martin R E, eds. Health effects of polyunsaturated fatty acids in seafoods. New York: Academic Press, 1986: 33

4 Corey E J, Shih C, Cash Man J R. Docosahexaenoic acid is a strong inhibitor of prostaglandin but not leukotriene biosynthesis. Proc Natl Acad Sci USA 1983; 80: 3581-4.

5 Needleman P, Raz A, Minkes M S, Ferrendelli J A, Sprecher H. Triene prostaglandins: prostacyclin and tromboxane biosynthesis and unique biological properties. Proc Natl Acad Sci USA 1979; 76: 944-8.

6 Terano T, Salmon J A, Moncada S. Biosynthesis and biologic activity of leukotriene B5. Prostaglandins 1984; 27: 217-32.

7 Strasser T, Fischer S, Weber P C. Leukotriene B5 is formed in human neutrophils after dietary supplementation with eicosapentaenoic acid. Proc Natl Acad Sci USA 1985; 82 $1540-3$

8 Allen R $\dot{C}$. Phagocytic leukocyte oxygenation activities and chemiluminescence: a kinetic approach to analysis. In: De Luca M A, McElroy W D, eds. Methods in enzymology. Vol. 33. Bioluminescence and chemiluminescence. New York. Academic Press, 1986: 449-93.

9 De Sole P, Lippa S, Littarru G P. Whole blood chemiluminescence: a new technical approach to assess oxygen nescence: a new technical approach to assess oxygen dependent microbicidal activity of granulocytes. Foum

10 Rossi $\mathrm{F}$. The $\mathrm{O}$ forming NADPH oxidase of the phagocytes: nature, mechanism of action and function. Biochem Biophys Acta 1986; 853: 65-89.

11 Magarò M, Altomonte L, Zoli A, et al. Influence of diet with different lipid composition on neutrophil chemiluminescence and disease activity in patients with rheumatoid arthritis. Ann Rheum Dis 1986; 47: 793-6.

12 Arnett F C, Edworthy S M, Bloch A D, et al. The American Rheumatism Association 1987 revised criteria for the classification of rheumatoid arthritis. Arthritis Rheum 1988; 31: $315-24$.

13 Robinson D R, Tateno S, Patel B, et al. The effects of dietary marine lipids on autoimmune diseases. In: Lanas W E M, ed. Polyunsaturated fatty acids and eicosanoids. Champain, ed. Polyunsaturated fatty acids and etcosanoids. Cham

14 Castagna M, Takai Y, Kaibuchi K, Sano K, Kikkawa V Nishizuka Y. Direct activation of calcium activated phos-
, pholipid-dependent protein kinase $C$ by tumor promoting phorbol esters. F Biol Chem 1982; 257: 7847-51.

15 Niedel J E, Kuhn L J, Vandenbark G R. Phorbol diester receptor copurifies with protein kinase C. Proc Natl Acad Sci USA 1983; 80: 36-40.

16 Ross E M. Signal sorting and amplification through $G$ protein-coupled receptors. Neuron 1989; 3: 14l-2.

17 Berridge M J. Inositol triphosphate and diacylglycerol as second messengers. Biochem $\mathcal{f}$ 1984; 220: 345-60.

18 Kremer J M, Jubiz W, Michalek A, et al. Fish oil fatty acid supplementation in active rheumatoid arthritis. Ann Intern Supplementation in active

19 Sperling R I, Weimblatt M, Robin J L, et al. Effect of dietary supplementation with marine fish oil on leukocyte lipid mediator generation and function in rheumatoid arthritis Arthritis Rheum 1987; 30: 988-97.

20 Van Der Tempel H, Tulleken J E, Limburg P C, Muskie F A J, Van Rijswijk M H. Effects of fish oil supplementation in rheumatoid arthritis. Ann Rheum Dis 1990; 49: 76-80.

21 Robinson D R, Corey E J, Lewis R A, Austen K F. Effect of dietary enrichment with eicosapentaenoic and docosahexaenoic acids on in vitro neutrophil and monocyte leukotriene generation and neutrophil function. $N$ Engl $\mathcal{f}$ Med 1985; 312: 1217

22 Goldman D W, Goetz E J. Human neutrophil chemotactic and degranulating activities of leukotriene B5 (LTB5) derived from eicosapentaenoic acid. Biochem, Biophys Res Commun 1983; 117: 282-8.

23 Lee T H. Effects of exogenous arachidonic eicosapentaenoic and docosahexaenoic acids on the generation of 5-lipoxygenase pathway products by ionophore activated human genase pathway products by ionophore acti
neutrophyls. $\mathcal{F}$ Clin Invest 1984; 74: 1922-33.

24 Hostmark A T, Bierkedal T, Kierulf $\mathrm{P}$, Flaten $\mathrm{H}$, Ulshagen K. Fish oil and plasma fibrinogen. $B M \mathcal{F} 1988 ; 297: 180-4$.
. 